\title{
ENSINO AO PACIENTE COM INSUFICIÊNCIA CARDÍACA: ESTRATÉGIAS UTILIZADAS NAS INTERVENÇÕES DE ENFERMAGEM
}

\author{
Cristina Silva Arruda ${ }^{1}$, Ana Carla Dantas Cavalcanti²
}

RESUMO: O objetivo deste estudo foi identificar estratégias de intervenção de enfermagem utilizadas no ensino do paciente com insuficiência cardíaca. Trata-se de uma revisão integrativa com abordagem quanti-qualitativa. Dos 270 artigos científicos encontrados 47 foram selecionados, $51 \%$ são estudos experimentais e observacionais, $25 \%$ revisões e $15 \%$ relatos de experiência, $57 \%$ de revistas americanas, $34 \%$ européias e $6 \%$ brasileiras. As estratégias mais utilizadas foram a discussão individual (47\%), monitorização telefônica (32\%) e uso de material escrito (25\%); estas foram desenvolvidas em 46\% dos casos em Clínicas de Insuficiência Cardíaca. Os estudos demonstram as ações direcionadas para o ensino e monitorização de pacientes e reflete a preocupação crescente na discussão de estratégias de intervenção com vistas a melhora na qualidade de vida dos pacientes com Insuficiência Cardíaca. Contudo, é necessário que as pesquisas contemplem a análise da eficácia da implementação das estratégias. PALAVRAS-CHAVE: Enfermagem; Insuficiência cardíaca; Educação.

\section{TEACHING THE PATIENT WITH CARDIAC INSUFFICIENCY: STRATEGIES USED IN NURSING INTERVENTIONS}

ABSTRACT: The aim of this study was to identify nursing intervention strategies used in the teaching of the patient with cardiac insufficiency. It is an integrative review with a quanti-qualitative approach. Of the 270 scientific articles found, 47 were selected, $51 \%$ are experimental and observational studies, $25 \%$ reviews and 15\% case reports. 57\% came from American magazines, $34 \%$ from European magazines, and 6\% from Brazilian magazines. The most-used strategies were individual discussion (47\%), telephone follow-up (32\%) and use of written material (25\%); these were developed in $46 \%$ of cases in Cardiac Insufficiency Clinics. The studies demonstrate the actions aimed at teaching and following up patients, and reflect the growing concern in discussion of intervention strategies which focus on the improvement of the quality of life of those patients with Cardiac Insufficiency. However, it is necessary for the research to consider the analysis of the effectiveness of implementation of the strategies.

KEYWORDS: Nursing; Cardiac insufficiency; Education.

\section{ENSEÑANZA AL PACIENTE CON INSUFICIENCIA CARDÍACA: ESTRATEGIAS UTILIZADAS EN LAS INTERVENCIONES DE ENFERMERÍA}

RESUMEN: El objetivo de este estudio fue identificar estrategias de intervención de enfermería utilizadas en la enseñanza del paciente con insuficiencia cardíaca. Es una revisión integrativa con abordaje cuanti-cualitativo. De los 270 artículos científicos encontrados 47 fueron seleccionados, 51\% son estudios experimentales y observacionales, $25 \%$ revisiones y $15 \%$ informes de experiencia, $57 \%$ de revistas americanas, $34 \%$ europeas y $6 \%$ brasileñas. Las estrategias más utilizadas fueron la discusión individual (47\%), monitorización telefónica (32\%) y uso de material escrito (25\%); estas fueron desarrolladas en $46 \%$ de los casos en Clínicas de Insuficiencia Cardíaca. Los estudios muestran las acciones direccionadas para la enseñanza y monitorización de pacientes y indican la preocupación creciente en la discusión de estrategias de intervención con fines de mejorar la cualidad de vida de los pacientes con insuficiencia cardíaca. Sin embargo, es necesario que las investigaciones contemplen el análisis de la eficacia de la implementación de las estrategias. PALABRAS CLAVE: Enfermería; Insuficiencia cardíaca; Educación.

${ }^{1}$ Enfermeira. Mestranda pelo Programa de Pós-Graduação em Ciências Cardiovasculares da Universidade Federal Fluminense - UFF. Membro do Grupo de Sistematização da Assistência de Enfermagem da UFF - GESAE.

${ }^{2}$ Enfermeira. Doutora em Enfermagem. Professora do Departamento de Fundamentos de Enfermagem da Escola de Enfermagem Aurora de Afonso Pinto da UFF. Líder do GESAE UFF.

Autor correspondente:

Recebido: 27/03/2011

Cristina Silva Arruda

Aprovado: 26/01/2012

Universidade Federal Fluminense

Rua Dr. Celestino, 74 - 24020-091 - Niterói-RJ-Brasil

E-mail: cris1486@gmail.com 


\section{INTRODUÇÃO}

Entre as principais doenças crônicas encontramos as cardiovasculares e, entre essas, a insuficiência cardíaca (IC), via final da maior parte dessas doenças, que atinge frequentemente pacientes com mais de 60 anos, sendo a principal causa de internação hospitalar, com 30 a $50 \%$ de readmissões em noventa dias ${ }^{(1)}$.

A IC é uma das maiores causas de incapacidade e morbidade, pois impossibilita a execução de atividades diárias e profissionais, além de predispor à alterações emocionais, como a depressão e a ansiedade. Traz impacto físico e psicológico sobre a própria existência e, assim, gera modificações sociais e nas atividades cotidianas ${ }^{(2)}$.

Vários estudos têm sido realizados para investigar as principais causas para o grande número de re-internações e mortalidade da IC. E entre estas, destacam-se o desconhecimento das medidas não-farmacológicas, a adesão inadequada ao tratamento medicamentoso e/ou a incapacidade dos pacientes de identificarem sinais e sintomas que antecedem a fase de descompensação ${ }^{(3-5)}$.

A terapêutica incentiva a identificação da causa principal da doença e seu tratamento ou remoção, a eliminação ou correção de fatores agravantes, modificações no estilo de vida e a atenção aos aspectos psicossociais ${ }^{(5)}$. Para tal, são estimuladas medidas não-farmacológicas de acompanhamento clínico e ensino do paciente sobre a doença, e seu tratamento por equipe multiprofissional, a fim de buscar a promoção destas mudanças e a adesão ao tratamento, diminuindo os índices de internação e mortalidade.

Daí surge a necessidade de conhecermos as intervenções de enfermagem utilizadas no Brasil e no mundo para pacientes com IC que alcançaram melhores resultados nesta adesão ao tratamento e consequente melhora na qualidade de vida, diminuição das hospitalizações.

Considerando que a meta para o controle e tratamento da IC é a adesão ao tratamento, o ensino do paciente deve ser a base das intervenções de enfermagem, incorporando o planejamento, implementação e avaliação de um programa elaborado para atender suas necessidades especiais ${ }^{(4)}$. Portanto, este estudo torna-se relevante por apresentar as diferentes estratégias de ensinar o paciente com IC a atingir modificações no estilo de vida, nos remetendo a reflexões sobre as variadas maneiras de fazer Enfermagem com o intuito de atingir melhores resultados frente à problemática instalada pela IC.

Assim, este estudo teve como objetivo identificar estratégias para o ensino como intervenção de enfermagem ao paciente com insuficiência cardíaca.

\section{MÉTODO}

Neste estudo foi realizada uma revisão integrativa. Para tal, foram adotadas seis etapas: seleção de uma questão para a revisão; escolha das pesquisas que participaram da amostra; representação das características da pesquisa revisada; análise dos achados de acordo com os critérios de inclusão e exclusão estabelecidos; interpretação e apresentação dos resultados ${ }^{(6-7)}$. As publicações foram encontradas a partir da busca nas bases eletrônicas de dados MEDLINE (Medical Literature Analysis and Retrieval System online) e LILACS (Literatura Latino-Americana e do Caribe em Ciências da Saúde), utilizando-se a terminologia em saúde consultada nos Descritores em Ciência da Saúde (DeCS/BVS): Insuficiência cardíaca (heart failure), Enfermagem (nursing) e Educação (education).

A busca ocorreu no segundo semestre de 2011 e janeiro de 2012, tendo como critérios para inclusão artigos publicados no período de 2000 a 2011 e que se relacionassem à intervenção de enfermagem à pacientes com insuficiência cardíaca; artigos repetidos foram considerados uma única vez. Foram excluídos desta revisão artigos que não estavam disponíveis em texto completo online, encontravam-se em outros idiomas que não o português ou inglês e/ou não descreviam estratégias de ensino utilizadas exclusivamente para pacientes com insuficiência cardíaca.

A partir de então os artigos selecionados foram examinados utilizando-se como diretriz um formulário previamente planejado e que continha: a) dados de identificação (título, autores, ano e país de publicação do periódico); b) objetivos do estudo; c) delineamento e/ou método de estudo; d) cenário de estudo; e) intervenção de ensino desenvolvida; f) banco de dados. Essas informações foram contidas em um banco de dados organizado no Programa Microsoft Excel do pacote Windows.

A análise dos dados foi feita pela leitura dos artigos, culminando em sua interpretação quantitativa de acordo com a frequência simples de estratégias de ensino, expressando suas informações através de tabelas e gráficos; e análise qualitativa, relevando sua aplicabilidade nos estudos.

\section{RESULTADOS}

A aplicação dos descritores Insuficiência Cardíaca, Educação e Enfermagem nas bases de dados selecionadas revelaram a existência de 270 artigos. 
Entretanto, aplicando-se os critérios de exclusão, foram selecionados 47 artigos para a construção desta revisão, distribuídos nos anos selecionados, de acordo com o apresentado no gráfico 1 .

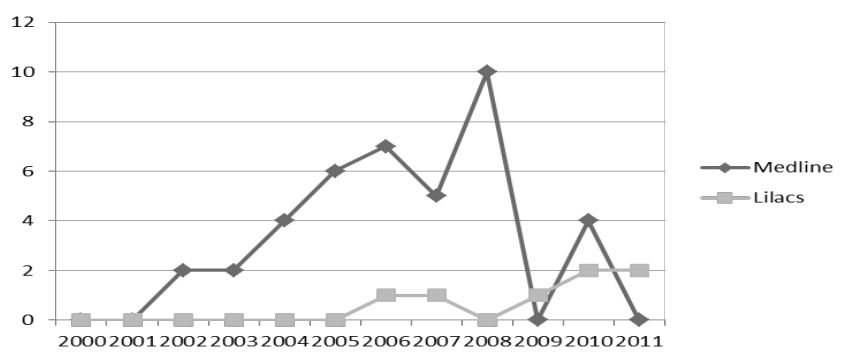

Gráfico 1- Número de artigos sobre estratégias de ensino a pacientes com insuficiência cardíaca de acordo com o ano de publicação ( $\mathrm{n}=45), 2000-2011$. Niterói, 2012

Das 24 revistas referidas nas 47 publicações, duas são brasileiras: Online Brazilian Journal of Nursing do estado do Rio de Janeiro e a Revista Latino-Americana de Enfermagem de São Paulo, totalizando 6\% $(n=3)$ das publicações. As de publicação americana formam $57 \%(n=27)$ do total, as européias 34\% $(n=16)$ e australiana 2\% (n=1). Porém, a revista European Journal Cardiovascular Nursing (européia) possui 25\% (n=12) dos artigos indexados, sendo a revista com o maior número de publicações, seguida pela Progress in Cardiovascular Nursing (americana) com $8 \%(n=4)$.

Com relação ao método, os artigos selecionados foram assim classificados: estudos experimentais e observacionais $51 \%(\mathrm{n}=24)$, revisão de literatura $25 \%$ $(n=12)$ e relatos de experiência $15 \%(n=7)$. E ainda $8 \%$ $(n=4)$ não especificaram o tipo de pesquisa.

Dos 47 artigos, 74\% $(n=35)$ descreveram algum cenário para suas intervenções tendo as clínicas de IC maior representatividade, sendo citadas em $46 \%(n=16)$ dos estudos, os hospitais foram citados em 17\% $(n=6)$. O cenário ambulatorial foi apontado tanto na Atenção Primária em Saúde, em 8\% (n=3), quanto nas Clínicas de Cardiologia, em 6\% ( $\mathrm{n}=2)$ dos estudos. A visita domiciliar foi referida $17 \%(n=6)$ como um cenário que facilita a abordagem dos pacientes e seus familiares. A comunidade foi o cenário utilizado nos estudos que discutem o manejo de populações específicas, apresentando frequência de $6 \%(\mathrm{n}=2)$.

Na maioria dos estudos foram estratégias de ensino a discussão individual, a monitorização telefônica e fornecimento de material escrito, como panfletos, cartazes e guia de instruções, e diários. As outras estratégias utilizadas foram grupo de orientação, ensino sobre exercício físico, utilização do vídeo, uso do computador com a página de web, CD-Rom e correio eletrônico, cuja distribuição percentual está apresentado no gráfico 2. Grande parte dos estudos não abordou apenas uma estratégia, integrando muitas vezes duas ou três delas.

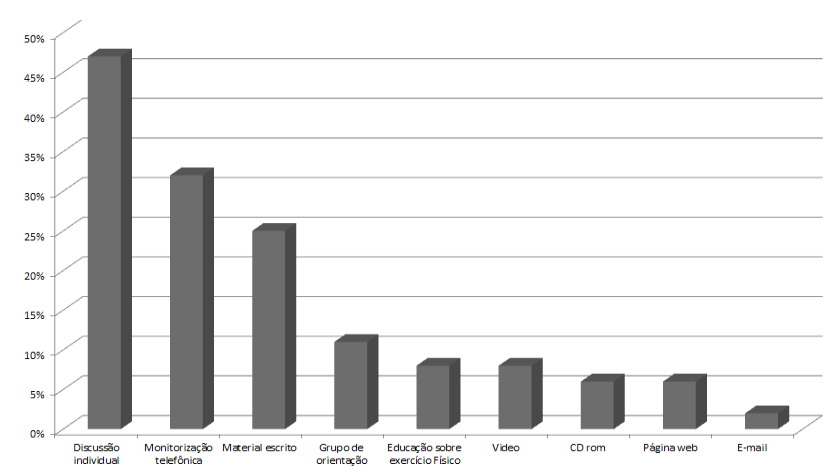

Gráfico 2- Frequência das estratégias de ensino abordadas nos estudos sobre estratégias de ensino a pacientes com insuficiência cardíaca de acordo com o ano de publicação $(\mathrm{n}=45), 2000-2011$. Niterói, 2012

A frequência das estratégias encontradas nos estudos foi: $47 \%(n=22)$ pela discussão individual; $32 \%(n=15)$ pela monitorização telefônica; o material escrito como panfletos, diários, cartazes e guia de instruções com $25 \%(\mathrm{n}=12)$; grupo de orientação em $11 \%(n=5)$; o ensino sobre exercício físico e a utilização do vídeo em $8 \%(n=4)$ cada; o uso do computador com a página de web em $6 \%(n=3), C D-R o m$ em $6 \%(n=3)$ e o correio eletrônico foi citado em $2 \%(n=1)$ dos estudos. Grande parte dos estudos não abordou apenas uma estratégia, integrando muitas vezes duas ou três delas.

\section{DISCUSSÃO}

A IC é uma doença de incidência mundial, no entanto, preocupa principalmente os países onde a população de idosos é crescente ou maior que as demais faixas etárias, já que o avanço no tratamento das doenças cardiovasculares propiciam a sobrevida e o aparecimento desse agravo à saúde ${ }^{(8)}$. Desta forma, a publicação direcionada a este assunto tem sido desenvolvida em diversos locais do mundo, mas se destaca o número de publicações na Europa e América do Norte, continentes com maior número de idosos e maiores recursos socioeconômicos, onde pesquisas epidemiológicas e experimentais estão sendo realizadas. O Brasil foi representado por apenas três estudos, evidenciando a necessidade de pesquisas nacionais que demonstrem resultados de centros científicos e evidenciem seus aspectos culturais. 
O crescente número de artigos publicados demonstra que a realização de novas investigações sobre o problema em estudo vem aumentando. Paralelamente, na última década, houve progressiva elevação do número de casos da doença e, consequentemente, de maiores custos, aguçando a necessidade da realização de novos estudos ${ }^{(1)}$.

Apesar deste estudo apontar 47 artigos sobre estratégias de ensino para o paciente com IC, uma das limitações foi não apresentar metodologias semelhantes que permitissem correlações entre os mesmos. No entanto, foi possível evidenciar que as estratégias apontadas não foram utilizadas apenas como meio de ensinar o paciente com IC a entender a doença, mas como forma de monitorização ativa de suas ações e do estado de saúde em que se encontra. A palavra monitorizar se refere ao acompanhamento e avaliação dos dados fornecidos. Ao considerarmos a extensão de informações e de cuidados que deve ser assimiladas pelos pacientes, deve-se ressaltar a importância desta ação para a garantia da adesão ao tratamento. ${ }^{(9)}$.

A monitorização desses pacientes deve servir não apenas como busca de erros na conduta terapêutica, mas também como incentivo e apoio. Muitos estudos demonstraram que em programas onde ocorre o acompanhamento intensivo por parte dos profissionais, os pacientes se tornam mais seguros e acabam cumprindo suas metas ${ }^{(10-12)}$. Assim, nos artigos que abordam formas de monitorização, as estratégias utilizadas foram: monitorização telefônica, discussão individual, material escrito e página da web, grupo de orientação e e-mail.

Ensinar é ministrar o ensino, lecionar ou transmitir $\operatorname{conhecimentos}^{(9)}$. Por isso, o enfermeiro face ao tratamento da IC deve capacitar pacientes e familiares, instruindo-os quanto às práticas que irão compor o cuidado diário, transmitindo os conhecimentos comprovados por estudos e lecionando através das diversas estratégias existentes sobre como este deve agir frente a uma situação nova ${ }^{(2,8,13)}$.

Dentre os estudos que utilizaram intervenções de enfermagem como formas de ensino, as estratégias empregadas foram: discussão individual, realizada no atendimento hospitalar e nas visitas domiciliares, materiais escritos, grupos de orientação, CD-Rom e vídeo, uso da página da $w e b$, conversa telefônica, além das informações sobre a construção de um plano de educação sobre exercício físico.

O método de ensino deve se basear na necessidade de aprendizagem do paciente e servir como veículo para que a informação seja transmitida e entendida ${ }^{(2,8)}$. As pessoas processam as informações de diversos modos como vendo e ouvindo, refletindo e agindo, raciocinando logicamente ou intuitivamente, analisando e visualizando. Por isto, os métodos de ensino devem explorar ao máximo todas essas formas, fazendo com que o aprendizado seja mais rico e completo ${ }^{(10)}$. Em conjunto a informação verbal, deve haver uma combinação de materiais educativos para que a capacidade de absorção das informações seja elevada. Livros, boletins informativos, DVD, CDs, páginas da Web, computadores e seus programas interativos em conjunto facilitam o processo de aprendizagem, pois abrangem uma oferta maior de novas oportunidades para o ensino.

Na discussão individual o monitoramento acontece a partir da troca de informações, possibilitando a avaliação do conhecimento adquirido. Essa é realizada através de uma simples conversa e pode utilizar materiais escritos, jogos e vídeos ${ }^{(10)}$. Além disso, a família pode ser incluída como forma de ajuda na absorção das ideias e como observação das atitudes adotadas no ambiente familiar ${ }^{(14)}$.

Em um dos estudos analisados as consultas de enfermagem eram adotadas como meio de discussão individual, sendo dividida em duas fases: a primeira de forma explicativa-informativa e a segunda com dicas de práticas cotidianas a partir de dificuldades encontradas por cada paciente e seus cuidadores ${ }^{(14)}$.

Cabe lembrar que a discussão com a clientela referida nos estudos analisados não foi utilizada como forma de repreensão, mas como oportunidade de orientação sobre hábitos nocivos. Podendo essa ser desenvolvida em vários cenários e ser estabelecida em qualquer contato com o paciente. Além disso, estimula o envolvimento e a reflexão do paciente que se sente instigado a buscar respostas ao seu problema e não só receber as informações do profissional de saúde. No entanto, o planejamento e o desenvolvimento de metas são ferramentas essenciais para o alcance da adesão ao tratamento ${ }^{(15)}$.

Os profissionais de saúde devem abordar o problema de forma indagadora, perguntando o que incomoda, ou seja, o que é difícil para o paciente com IC, o que o deixa mais angustiado e o que deseja alterar, identificando o que realmente é importante e direcionando as intervenções de enfermagem ${ }^{(16)}$.

A estratégia de ensino por meio de grupos de orientação traz a oportunidade de interação entre os pacientes que compartilham vivências semelhantes ${ }^{(7)}$. Com a possibilidade do desenvolvimento de metas e reflexão dos hábitos de vida que não se encerra apenas com a discussão entre paciente e profissional, mas se estende 
aos demais indivíduos constituintes do grupo ${ }^{(15)}$.

Estes podem opinar e diferenciar em pontos de vista mesmo que estejam vivendo a mesma situação. Isto enriquece a dinâmica e possibilita que diversas soluções para os problemas surjam, trazendo uma nova forma de apoio e de intervenção motivacional ${ }^{(17)}$.

Os estudos indicam a formação de grupos de orientação de pacientes com IC, com discussão direta de casos pessoais e promoção do apoio para o estabelecimento da auto-gestão do tratamento. Os próprios pacientes elencavam fatores em desacordo com o seu tratamento e, juntamente com o enfermeiro, traçavam metas e estratégias para melhoria da qualidade de $\mathrm{vida}^{(11,15)}$. Em um dos estudos ${ }^{(15)}$, em uma escala de 1 a 5, os pacientes atribuíram média 4,04 para a qualidade das sessões de grupo, e em outro ${ }^{(11)} 75 \%$ dos participantes melhoram seu conhecimento para o autocuidado.

Os estudos indicam que ao iniciar um grupo deve-se deixar claro aos participantes o objetivo pelo qual ele foi montado, suas metas, possível duração e as estratégias que serão empregadas. Desta forma, os interessados podem avaliar a sua real possibilidade de participação e esclarecer dúvidas. Também não devem ser constituídos grupos numerosos e nem muito pequenos, para que não se corra o risco de uma alta rotatividade entre os participantes, permeando o número de 15 integrantes. Deve haver pelo menos um líder, que ajudará na discussão dos assuntos e representará as partes interessadas ${ }^{(10,15)}$.

No resgate das informações foram apresentados os materiais escritos. Estes são formas simples de registrar as orientações fornecidas, promovendo a revisão e exercício da leitura e escrita ${ }^{(17)}$. Destaca-se o uso desses com pacientes que apresentam déficit de memória. E, entre as variadas formas de apresentação, pode-se citar como exemplos, os gráficos de peso, folhetos, cartazes, diários, lista de temperos e de supermercado e orientações de medicamentos ${ }^{(18)}$.

O grupo focal foi apresentado como estratégia de ensino para mulheres idosas portadoras de IC com dificuldades de seguir uma dieta hipossódica. Junto ao grupo, foi utilizado um guia de orientações escritas sobre alimentação que eram reforçadas pelo enfermeiro para o controle da dieta e dos níveis de pressão arterial das participantes ${ }^{(19)}$.

A utilização de material escrito, como a tabela esquemática com nome e horário das medicações foi apresentada em um estudo com 60 pacientes com IC, conseguindo diminuir os índices de omissão das doses dos medicamentos em seus horários corretos e aumentando o conhecimento sobre sua indicação e de efeitos colaterais em $80 \%{ }^{(20)}$.

Outra forma de contribuição, ou até de introdução, de um novo conteúdo é a estratégia do vídeo. Este pode ser adotado durante a hospitalização, no acompanhamento ambulatorial e/ou em domicílio, devendo sempre abordar assuntos do cotidiano desses pacientes. $\mathrm{O}$ vídeo foi utilizado como estratégia de ensino para a promoção de educação intensiva em paciente de difícil aderência ao tratamento; foram vistos durante a internação e entregues após a alta e demonstrou melhora na adesão terapêutica ${ }^{(21)}$.

A supervisão constante das ações desses pacientes também pode ser conseguida através da monitorização telefônica, possibilitando a introdução das informações de acordo com as dificuldades encontradas pelas pessoas, atendendo um caráter mais individual e objetivo. Esta serve, portanto, como complementação de uma estratégia de ensino mais abrangente, já que deve estar ligada sempre a uma atividade presencial ${ }^{(8)}$. Esta estratégia foi utilizada no acompanhamento pós-alta hospitalar, após realização de outras estratégias de educação, como o grupo de orientação e a consulta de enfermagem, e mostraram melhores resultados do que nos grupos onde o acompanhamento telefônico não foi inserido $^{(22-23)}$. $\mathrm{O}$ acompanhamento intensificado por profissionais de saúde promove conforto emocional, já que o paciente sabe onde pode procurar ajuda, sendo muito importante, principalmente, no período pós-alta hospitalar, em que a memorização do plano de cuidados ainda é deficiente e as dúvidas são mais constantes ${ }^{(10)}$.

Como meios contemporâneos para o estabelecimento de informações utilizou-se o CD-ROM, a página da web, o telemonitoramento e o e-mail. Estes introduzem o uso do computador nas formas de ensino e promovem maior autonomia ${ }^{(4)}$. Apesar das dificuldades, estas estratégias não devem ser restritas aos jovens, e são profundamente eficazes em pessoas com baixa escolaridade. Essas permitem a possibilidade de manipular o conteúdo programado na rapidez e no momento que se sentirem mais confortáveis, promovendo a assimilação das informações no tempo do paciente e não em algo préfixado, além de disponibilizarem informações de maneira contínua, promovendo a combinação de diversas estratégias.

Além disso, algumas ferramentas oferecem um canal aberto para a retirada de dúvidas e questionamentos. Para que estas estratégias sejam ainda mais interessantes, demonstrou-se que devem ser construídas em parceria, profissional - paciente $^{(4)}$. Outra vantagem é a combinação de diversas técnicas de ensino, como, materiais escritos, vídeos e fotografias. 
No uso de uma página da web direcionada a pacientes idosos e seus cuidadores, as informações foram disponibilizadas em três módulos no período de 12 semanas, sendo incluído uma rede de conversação entre usuários e um correio eletrônico com a equipe de enfermeiros, conseguiu-se comprovar melhora na qualidade de vida e na capacidade de auto-gestão do tratamento destes pacientes quando comparados aos que sofreram intervenção de ensino padrão ${ }^{(24)}$.

Ainda como estratégia de ensino, os estudos destacaram o caráter ímpar das atividades físicas. Este assunto deve ser abordado de forma cuidadosa e individual, já que o estabelecimento de um programa de exercícios deve respeitar as características de cada paciente e deve sofrer monitorização constante ${ }^{(24)}$.

Os dados pesquisados não sugeriram as estratégias mais eficientes para a melhora da adesão ao tratamento. No entanto, ressalta-se a necessidade da identificação junto com o paciente da estratégia que apresenta maior viabilidade e demonstra melhores possibilidades de atingir resultados propostos.

\section{CONCLUSÃO}

Este estudo identificou as principais estratégias de ensino como intervenções de enfermagem para o paciente com IC e como estas podem ser adotadas pelos estabelecimentos de saúde e profissionais; poucos apresentaram a eficácia da implementação, sendo relevante estudos que discutam esse aspecto objetivando o alcance da meta de ensino e as particularidades do público-alvo.

A intervenção mais valorizada foi a discussão individual com o paciente, que apesar de fundamental, deve estar inserida juntamente com o desenvolvimento de atividades lúdicas que facilitem o aprendizado desta clientela.

$\mathrm{O}$ aumento progressivo de publicações demonstrou que a compreensão dos fenômenos correntes no processo de ensino é importante para que seja alcançada a adesão ao tratamento. Tornando-se necessário a realização de estudos que discutam a eficácia de cada uma das propostas para o alcance da meta de ensino e as particularidades de seu público.

\section{REFERÊNCIAS}

1. Ministério da Saúde (BR). Departamento de Informática do SUS/Informações de Saúde/ Informações Epidemiológicas e Morbidade/ Morbidade Hospitalar do SUS. [acesso em 10 mar 2009]. Disponível: http:/ tabnet.datasus.gov.br/cgi/deftohtm.exe?sih/cnv/niuf.def

2. Strömberg A. Educating nurses and patients to manage heart failure. Eur. J. Cardiovasc. Nurs. 2002;1:33-40.

3. Rabelo ER, Aliti GB, Goldraich L, Domingues FB, Clausell $\mathrm{N}$, Rohde LE. Manejo não-farmacológico de pacientes hospitalizados com insuficiência cardíaca em hospital universitário. Arq. Bras. Cardiol. 2006;87(3):352-8.

4. Cavalcanti ACD, Correia DMS, Queluci GC. A implantação da consulta de enfermagem ao paciente com insuficiência cardíaca. Rev. Eletr. Enf. [Internet] 2009;11(1):194-9.

5. SBC. III Diretrizes da Sociedade Brasileira de Cardiologia para o diagnóstico e tratamento da insuficiência cardíaca. Arq. Bras. Cardiol. 2009;79 Suppl IV:1-30.

6. Mendes KDS, Silveira RCCP, Galvão CM. Revisão integrativa: método de pesquisa para a incorporação de evidências na saúde e na enfermagem. Texto Contexto Enferm. 2008;17(4):758-64.

7. Vaz DC, Silva CRL, Figueiredo NMA. A utilização da bibliometria na análise do referente conforto. Cogitare enferm. 2010;15(4):736-41.

8. Yera-Casas AM, Mateos-Higuera Del Olmo S, Ferrero-Lobo J, Paez-Gutierrez TD. Evaluation of an educational intervention in elderly patients with heart failure performed by nurses using a standardized care plan. Enferm Clin. 2009;19(4):191-8.

9. Ferreira, $\mathrm{ABH}$. Minidicionário da língua portuguesa. $3^{a}$ ed. Rio de Janeiro: Nova Fronteira; 1993.

10. Fredericks S, Beanlands H, Spalding K; Silva M. Effects of the characteristics of teaching on the outcomes of heart failure patient education interventions: a systematic review. Eur. J. Cardiovasc. Nurs. 2010;9(1):30-7.

11. Lupón J, González B, Mas D, Urrutia A, Arenas M, Domingo M, et al. Patients' self-care improvement with nurse education intervention in Spain assessed by the european heart failure self-care behaviour scale. Eur. J. Cardiovasc. Nurs. 2008;7(1):6-20.

12. Sisk JE, Hebert PL, Horowitz CR, McLaughlin MA, Wang JJ, Chassin MRl. Effects of nurse management on the quality of heart failure care in minority communities: a randomized trial. Ann. internal med. 2006;145(4):273-83. 
13. Wheeler E, Plowfield L. Clinical education initiative in the community: caring for patients with congestive heart failure. Nurs. educ. perspect. 2004;25(1):16-21.

14. Mariani M, Vella G, Bianchi C, Verde S, Maria R, Pirrelli S. Implementation of beta-blockade in elderly heart failure patients: role of the nurse specialist. Eur. J. Cardiovasc. Nurs. 2008;7(3):196-203.

15. Schreurs KM, Colland VT, Kuijer RG, de Ridder DT, Van Elderen T. Development, content and process evaluation of a short self-management intervention in patients with chronic diseases requiring self-care behaviours. Patient Educ. Couns. 2003;51(2):133-41.

16. Huffman M. Health Coaching: a new and exciting technique to enhance patient self-management and improve outcomes. Home health. nurse. 2007;25(4):271-4.

17. Washburn SC, Hornberger CA. Nurse educator guidelines for the management of heart failure. J. Contin. Educ. Nur. 2008;39(6):263-7.

18. Rabelo ER, Aliti GB, Domingues FB, Ruschel KB, Brun AO. O que ensinar aos pacientes com insuficiência cardíaca e porquê: o papel dos enfermeiros em clínicas de insuficiência cardíaca. Rev. Latino-Am. Enfermagem. 2007;15(1):165-70.

19. Sheahan SL, Fields B. Sodium dietary restriction, knowledge, beliefs and decision-making behavior of older females. JAANP. 2008;20(4):217-24.

20. Crowther M. Optimal management of outpatients with heart failure using advanced practice nurses in HospitalBased Heart Failure Center. JAANP. 2003;15(6):260-5.

21. Kodiath M, Kelly A, Shively M. Improving quality of live in patients with heart failure: an innovative behavioral intervention. J Cardiovasc Nurs. 2005;20(1):43-8.

22. Kutzleb J, Reiner D. The impact of nurse-directed patient education on quality of life and functional capacity in people with heart failure. JAANP. 2006;18(3):116-23.

23. Westlake C, Evangelista LS, Strömberg A, Tergalstanyan A, Vazirani S, Dracup K. Evaluation of web-based education and counseling pilot program for older heart failure patients. Prog Cardiovasc Nurs. 2007;22(1):20-6.

24. Johansson P, Dahlström U, Broström A. Factors and interventions influencing health-related quality of life in patients with heart failure: a review of the literature. Eur. J. Cardiovasc. Nurs. 2006;5(1):5-15. 